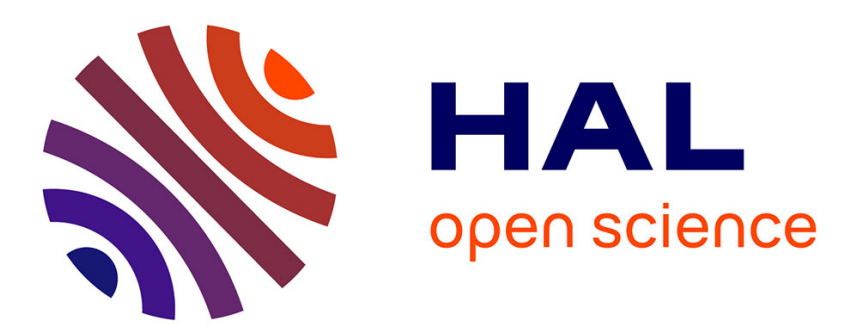

\title{
Connaissances et besoins de formation des enseignants du second degre concernant les Troubles du spectre de l'autisme
}

\author{
Eric Flavier, Céline Clément
}

\section{- To cite this version:}

Eric Flavier, Céline Clément. Connaissances et besoins de formation des enseignants du second degre concernant les Troubles du spectre de l'autisme. La nouvelle revue de l'adaptation et de la scolarisation, 2013, 65, pp.1-18. hal-01070564

\section{HAL Id: hal-01070564 \\ https://hal.science/hal-01070564}

Submitted on 1 Oct 2014

HAL is a multi-disciplinary open access archive for the deposit and dissemination of scientific research documents, whether they are published or not. The documents may come from teaching and research institutions in France or abroad, or from public or private research centers.
L'archive ouverte pluridisciplinaire HAL, est destinée au dépôt et à la diffusion de documents scientifiques de niveau recherche, publiés ou non, émanant des établissements d'enseignement et de recherche français ou étrangers, des laboratoires publics ou privés. 


\title{
Connaissances et besoins de formation des enseignants du second degré concernant les Troubles du Spectre de l'Autisme
}

\author{
Eric FLAVIER ${ }^{1} \&$ Céline CLEMENT ${ }^{2}$
}

1 - Ecole Supérieure du Professorat et de l'Education et LISEC EA 2310, Université de Strasbourg, eric.flavier@unistra.fr

2 - Ecole Supérieure du Professorat et de l'Education et LISEC EA 2310, Université de Strasbourg, celine.clement@unistra.fr

Cette recherche a été financée par le Conseil Scientifique de l’Université de Strasbourg et l'IUFM d'Alsace

Correspondance pour cet article

Eric FLAVIER

ESPE de l'académie de Strasbourg

141 avenue de Colmar

BP 40102

67024 STRASBOURG Cedex

eric.flavier@unistra.fr 


\title{
Connaissances et besoins de formation des enseignants du second degré concernant les Troubles du Spectre de l'Autisme
}

\begin{abstract}
Résumé
L'augmentation relative du nombre d'élèves du second degré présentant des Troubles du Spectre de l'Autisme (TSA) impose de s'interroger sur les connaissances des enseignants concernant les TSA et d'identifier leurs besoins de formation. Pour ce faire, notre étude propose un cadre méthodologique croisant des données quantitatives et qualitatives. Les résultats de cette étude exploratoire mettent en évidence : le faible niveau de connaissances des enseignants concernant l'intervention auprès des élèves présentant un TSA ; l'estimation des besoins de formation positivement corrélée au retentissement supposé en classe; le caractère singulier et situé des connaissances mobilisées en classe. Les résultats sont discutés dans le sens d'une réflexion sur les nécessités inhérentes à la formation des enseignants accueillant des élèves avec TSA.
\end{abstract}

Mots clés : autisme - inclusion - besoin de formation - enseignants - second degré

\section{Summary}

The increasing number of high school children with autism spectrum disorders (ASD) requires questioning teachers' knowledge about ASD and identifying their training needs. For this purpose, our study provides a methodological framework mixing quantitative and qualitative data. The findings from this exploratory study highlight the following: the low level of teachers' knowledge regarding intervention for students with ASD; the estimated training needs positively correlated with the expected impact in the classroom; the singular and situated nature of knowledge used in the classroom. The results are discussed toward a reflection on the inherent requirements of the training program for teachers with ASD students.

Keywords: autism - inclusion - training needs - teachers - high school un trouble du spectre de l'autisme. Nouvelle Revue de l'Adaptation et de la Scolarisation, 65, 95-112. 
Les Troubles du Spectre de l'Autisme (TSA) sont définis comme « un groupe de troubles caractérisés par des altérations qualitatives des interactions sociales réciproques et des modalités de communication, ainsi que par un répertoire d'intérêts et d'activités restreints, stéréotypés et répétitifs. Ces anomalies qualitatives constituent une caractéristique envahissante du fonctionnement du sujet, en toute situation » (Organisation Mondiale de la Santé, 1994).

La prévalence importante des TSA, estimée à 1 naissance sur 150 (Haute Autorité de Santé, 2012), induit des changements importants des politiques publiques. Ainsi trois plans autisme se succèdent depuis 2005, la Haute Autorité de Santé (HAS) a publié des recommandations quant au diagnostic et à l'accompagnement des personnes avec autisme (HAS, 2012) et l'autisme a été décrété « grande cause nationale » (Ensemble pour l'autisme, 2012).

Par ailleurs la «Loi pour l'égalité des droits et des chances, la participation et la citoyenneté des personnes handicapées » du 11 février 2005, qui fait de l'inclusion en milieu scolaire ordinaire un droit, pose de nouvelles questions aux professionnels de l'éducation. En effet, on assiste à une augmentation du nombre d'enfants présentant un TSA en milieu scolaire ordinaire; ils seraient ainsi un peu plus de 20000 en milieu ordinaire, majoritairement en primaire selon le Comité Economique, Social et Environnemental(CESE, 2012). Le même CESE évoque 1761 élèves souffrant d'autisme ou de troubles envahissants du développement scolarisés en Unités Localisées pour l'Inclusion Scolaire (ULIS). Ceci étant, le droit à l'éducation reconnu n'est pas accordé à tous les enfants avec autisme, que celui-ci soit donné à l'école ordinaire ou en établissement spécialisé. Un rapport de deux sénatrices (Campion et Debré, 2012) avance le nombre de 20000 enfants non scolarisés parmi lesquels les polyhandicapés et les autistes sévères avec retard mental.

Les modalités et obstacles de l'inclusion au primaire sont bien documentés (p.ex. Paquet, Clément et Magerotte, 2012). Ainsi, en France comme à l'étranger, l'une des questions soulevées concerne les besoins de formation des enseignants pour l'accueil des enfants et adolescents. Dans une étude réaliseee par Helps, Newson-Davis et Callias (1999), alors qu'ils étaient $70 \%$ à accueillir des enfants avec autisme, les enseignants et employés de soutien (équivalents aux auxiliaires de vie scolaire en France) ont énoncé des besoins de formation importants, allant jusqu'à demander n'importe quelle formation pour $37 \%$ d'entre eux. Plus intéressant encore, Helps et al. (1999) indiquent que les enseignants ont tendance à surestimer les capacités cognitives des enfants avec TSA, et que l'autisme est considéré comme un trouble émotionnel plutôt qu'un trouble du développement. En conséquence, le manque de connaissances, lié à un manque d'information et de formation, engendre des stratégies pédagogiques inadaptées.

En revanche, peu de travaux ce sont intéressés à la problématique de l'inclusion au secondaire. L'étude québécoise de Guimond et Forget (2010) menée auprès d'enseignants du secondaire fait exception. Cette étude a montré que les besoins de formation énoncés par les enseignants étaient moins importants que dans le primaire. Les enseignants ont été interrogés sur leurs connaissances concernant des thématiques en lien avec l'autisme, leurs besoins de formation perçus sur ces thématiques et les retentissements supposés de ces thématiques en classe. Il apparaît que les besoins de formation énoncés ne sont pas corrélés à leur niveau de connaissances, mais avec le niveau de répercussions anticipé des thématiques en classe. Or, la principale condition de réussite de l'intégration d'adolescents ayant, par exemple un syndrome d'Asperger, est la connaissance de ce handicap par les professionnels (Juhel, 2005). Ceci apparaît d'autant plus problématique que le manque de formation des enseignants du Flavier, E., Clément, C. (2014). Besoins de formation des enseignants du second degré pour l'inclusion des élèves avec un trouble du spectre de l'autisme. Nouvelle Revue de l'Adaptation et de la Scolarisation, 65, 95-112. 
second degré et de l'ensemble des professionnels de l'éducation concernant l'autisme est important (Beauguitte, 2006).

\section{Objectifs de recherche}

Actuellement en France, au collège et au lycée, sont scolarisés principalement des adolescents ne présentant pas de déficience intellectuelle associée à l'autisme, mais des particularités cognitives et des difficultés dans les interactions sociale ou présentant le syndrome d'Asperger (autisme sans retard cliniquement significatif du langage ou du développement cognitif). Cependant ils présentent des caractéristiques spécifiques (p.ex. difficultés dans les interactions sociales, incompréhension du langage imagé et des coutumes sociales) qui nécessitent qu'ils soient accompagnés de façon adéquate pour leur intégration. Les enseignants sont en première ligne pour réaliser l'intégration. En ce sens, des préconisations ont été élaborées. Il s'agit par exemple d'instituer, dans la formation initiale des enseignants, un stage d'immersion auprès d'enseignants spécialisés ou un module d'enseignement spécifique sur les Troubles Envahissants du Développement (TED) et les troubles associés (Campion et Debré, 2012). Cependant, cette démarche n'apparait pertinente qu'à la condition que cette formation réponde aux besoins effectivement rencontrés sur le terrain. Or, très peu de travaux ont été menés sur cette question, en particulier dans le secondaire.

Ainsi, cette étude est élaborée en réponse à la mise en œuvre des volets successifs du Plan Autisme. Plus particulièrement, elle s'inscrit dans la lignée des quatre mesures suivantes du deuxième plan autisme 2008-2010 (Ministère des Affaires Sociales et de la Santé, 2008) : Mesure 6 ; actualiser et développer les contenus de la formation initiale des professionnels ; Mesure 7 : développer la formation tout au long de la vie professionnelle ; Mesure 8 : Faire évoluer les métiers; Mesure 9 : Elaborer des recommandations de pratique professionnelle et évaluer leur mise en œuvre.

Plus précisément cet article présente une recherche exploratoire qui vise à l'identification des besoins de formation des enseignants du second degré par la confrontation entre les besoins perçus qu'ils déclarent et les besoins réels qui émergent de l'analyse de leur pratique professionnelle.

\section{Méthode}

L'étude propose un cadre méthodologique croisant des données quantitatives et qualitatives, en s'inspirant du paradigme des «mixed methods research» (Johnson \& Onwuegbuzie, 2004). Ce paradigme a été éprouvé positivement dans d'autres études portant sur la scolarisation d'élèves avec autisme (Dillon \& Underwood, 2012). Une telle démarche de recherche, développée selon une approche concourante, reposant sur un traitement simultané de données quantitatives et qualitatives, favorise le travail de triangulation et de confrontation des apports issus de chacune des deux méthodes et vise à dépasser leurs limites respectives.

\section{Participants}

Vingt-sept personnes ont répondu à l'enquête par questionnaire. Ces répondants ont été contactés essentiellement via les réseaux sociaux sur l'ensemble du territoire français. Un formulaire de consentement à signature électronique précédait le questionnaire en lui-même. Les données de l'une d'entre elles ont été exclues car elle était en formation en master, sans poste dans le secondaire. De même, deux enseignants ayant indiqué avoir un enfant avec TSA ont renseigné la $3^{\text {ème }}$ partie consacrée aux difficultés rencontrées pour l'accueil en classe, Flavier, E., Clément, C. (2014). Besoins de formation des enseignants du second degré pour l'inclusion des élèves avec un trouble du spectre de l'autisme. Nouvelle Revue de l'Adaptation et de la Scolarisation, 65, 95-112. 
alors même qu'elles n'ont pas accueilli d'enfant dans leur propre classe. Compte tenu du fait que ces enseignants-parents ont considéré avoir des compétences spécifiques concernant l'inclusion d'élèves avec autisme qu'ils n'ont eux-mêmes pas pratiquée, leurs données ne sont pas retenues.

Les résultats présentés concernent donc 24 enseignants. Il s'agit de 7 hommes et 17 femmes ; 12 sont titulaires d'un CAPES (Certificat d'aptitude au professorat de l'enseignement du second degré), 1 d'un CAPET (Certificat d'aptitude au professorat de l'enseignement technique), 7 de l'agrégation, 1 du concours de Conseiller Principal d'Education et un dernier a obtenu le Concours recrutement de professeur des écoles. Treize enseignent en collège et onze en lycée général et technologique. Quatre de ces enseignants, deux hommes et deux femmes, ont accueilli au moins un enfant avec un TSA dans leur classe. Il est intéressant de noter que ces derniers enseignent en $6^{\text {ème }}$ au collège, soit la première classe dú secondaire. L'un d'entre eux indique s'occuper de l'intégration d'un élève en ULIS (Unité Localisée pour l'Inclusion Scolaire).

Simultanément, la partie qualitative de l'étude s'est déroulée en collaboration avec deux enseignants (dénommés Mary et Max dans le présent article). Ils ne faisaient pas partie de l'échantillon des participants répondant au questionnaire du volet quantitatif de l'étude, ceci afin d'éviter toute influence réciproque entre les deux démarches.Par ailleurs, le fait de porter la focale d'analyse sur la pratique " au quotidien » des enseignants implique de construire avec eux une relation de confiance selon une démarche singulière à l'image des études de cas ou des témoignages d'expérience. Les deux enseignants étaient titulaires du CAPA-SH (Certificat d'aptitude professionnelle pour les aides spécialisées, les enseignements adaptés et la scolarisation des élèves en situation de handicap), exerçaient en ULIS et accueillaient respectivement 10 et 3 élèves présentant des TSA. Leur engagement a reposé sur le volontariat et l'acceptation des conditions de la collaboration professionnel-chercheur.

\section{Recueil et traitement des données}

Trois types de données ont été recueillis pour cette étude, des données quantitatives via un questionnaire, des données qualitatives via des observations de séquences d'enseignement et des entretiens semi-directifs. Afin de faciliter la compréhension de la recherche, seront présentés successivement le recueil et le traitement des données quantitatives puis qualitatives.

\section{Données quantitatives}

Les données quantitatives ont été recueillies via un questionnaire. Celui-ci est une adaptation française d'un questionnaire américain le Needs assessment of special educators who serve students with autism, utilisé antérieurement par Hendricks (2007) dans sa forme originale et dans une première adaptation francophone par Guimont et Forget (2010). Ce questionnaire mesure de façon auto-rapportée, le niveau des connaissances des enseignants sur des thématiques relatives aux TED, les répercussions potentielles en classe de ces thématiques et leurs besoins de formation sur ces thématiques.

Il est constitué de deux parties pour tous les répondants. La première partie porte sur des caractéristiques socio-biographiques (sexe, diplôme, etc.) et la deuxième partie aborde neuf thématiques caractéristiques des TSA (Tableau 1). Sous forme d'échelle trois composantes sont mesurées pour chaque thématique. Soit le niveau de connaissances du répondant, le besoin d'obtenir de la formation et la répercussion potentielle de la thématique en classe. Les coefficients de cohérence interne pour notre étude sont respectivement de 0,90 (connaissances), 0,98 (besoin de formation) et 0,95 (répercussion potentielle en classe). Pour Flavier, E., Clément, C. (2014). Besoins de formation des enseignants du second degré pour l'inclusion des élèves avec un trouble du spectre de l'autisme. Nouvelle Revue de l'Adaptation et de la Scolarisation, 65, 95-112. 
le niveau des connaissances, l'échelle s'échelonne de 1 (peu de connaissances) à 5 (beaucoup de connaissances). Pour les besoins de formation, l'échelle s'échelonne de 1 (besoin minime de formation) à 5 (besoin majeur de formation). Pour les répercussions potentielles des thématiques en classe, l'échelle s'échelonne de 1 (peu de répercussions) à 5 (beaucoup de répercussions).

Une troisième partie du questionnaire est réservée aux enseignants ayant accueilli un élève avec autisme et vise à mieux cerner les difficultés spécifiques rencontrées.

Tableau 1: Caractéristiques des TED et énoncés du questionnaire correspondant dans la deuxième partie du questionnaire (d'après Guimond et Forget, 2010).

\begin{tabular}{|c|c|}
\hline Caractéristiques & Enoncés du questionnaire \\
\hline $\begin{array}{l}\text { Différences entre les } \\
\text { différents diagnostics de } \\
\text { TED }\end{array}$ & $\begin{array}{l}\text { Les caractéristiques et les différences entre chacun des TED : } \\
\text { autisme, troubles envahissants du développement non spécifié, } \\
\text { syndrome d'Asperger, syndrome de Rett et syndrome désintégratif } \\
\text { de l'enfance }\end{array}$ \\
\hline $\begin{array}{l}\text { Critè } \\
\text { du D }\end{array}$ & $\begin{array}{l}\text { Les caractéristiques des TED telles que définies par la plus version } \\
\text { du Manuel Diagnostic et Statistique des Troubles Mentaux (DSM- } \\
\text { IV-TR) }\end{array}$ \\
\hline $\begin{array}{l}\text { Caracté } \\
\text { compor }\end{array}$ & $\begin{array}{l}\text { L'étendue des caractéristiques comportementales associées aux } \\
\text { TED, incluant les comportements répétitifs, les intérêts restreints } \\
\text { et les comportements d'autostimulation. }\end{array}$ \\
\hline $\begin{array}{l}\text { Trou } \\
\text { assoc }\end{array}$ & $\begin{array}{l}\text { Les troubles médicaux et physiologiques associés aux TED, } \\
\text { incluant les troubles convulsifs, les infections de l'oreille, les } \\
\text { troubles gastro-intestinaux, les troubles alimentaires et les troubles } \\
\text { du sommeil. }\end{array}$ \\
\hline $\begin{array}{l}\text { Trou } \\
\text { comi }\end{array}$ & $\begin{array}{l}\text { Les différents troubles de la communication associés aux TED, } \\
\text { incluant la présence d'écholalie, des déficits dans le langage } \\
\text { expressif, dans la compréhension du langage et dans les aptitudes } \\
\text { requises pour maintenir une conversation. }\end{array}$ \\
\hline $\begin{array}{l}\text { Problèmes liés } \\
\text { l'apprentissage }\end{array}$ & $\begin{array}{l}\text { Les problèmes d'apprentissage associés aux TED, incluant les } \\
\text { difficultés d'attention, d'organisation, de récupérer de } \\
\text { l'information, d'inférences et de résolution de problèmes. }\end{array}$ \\
\hline $\begin{array}{l}\text { Hypo et hyp } \\
\text { sensibilité }\end{array}$ & $\begin{array}{l}\text { Les différents patrons de réactions aux stimulations sensorielles } \\
\text { associées aux TED, incluant l'hypo et l'hypersensibilité aux } \\
\text { stimulations : visuelles, auditives, alimentaires, olfactives, tactiles, } \\
\text { proprioceptives et vestibulaires. }\end{array}$ \\
\hline $\begin{array}{l}\text { Déficit dans les } \\
\text { interactions sociales }\end{array}$ & $\begin{array}{l}\text { L'étendue des déficits dans les interactions sociales associées aux } \\
\text { TED, incluant les déficits d'attention envers les autres, d'attention } \\
\text { conjointe, d'imitation, de communication non-verbale, } \\
\text { d'expression émotionnelle et de compréhension de la perspective } \\
\text { d'autrui. }\end{array}$ \\
\hline $\begin{array}{l}\text { Problèmes } \\
\text { psychologiques }\end{array}$ & $\begin{array}{l}\text { Les différents problèmes psychologiques associés aux TED, } \\
\text { incluant la dépression, la solitude, le sentiment d'isolement, la } \\
\text { baisse de l'estime de soi et le rejet par les pairs. }\end{array}$ \\
\hline
\end{tabular}

Flavier, E., Clément, C. (2014). Besoins de formation des enseignants du second degré pour l'inclusion des élèves avec un trouble du spectre de l'autisme. Nouvelle Revue de l'Adaptation et de la Scolarisation, 65, 95-112. 
Les données du questionnaire ont été recueillies via la plateforme googledrive ${ }^{\circledR}$ et traitées avec le logiciel statistica $7 \circledR$. Selon les objectifs des comparaisons statistiques des tests $t$ pour échantillons appariés et des analyses de corrélation ont été effectuées.

\section{Données qualitatives}

Trois séquences d'enseignement menées par Mary $(n=2)$ et Max $(n=1)$ ont fait l'objet d'observations non participantes par l'un des chercheurs. Elles avaient pour objectif de permettre au chercheur de se familiariser avec le contexte d'exercice professionnel des deux enseignants afin de l'aider dans la conduite des entretiens. Plus précisément, les observations ont permis au chercheur de repérer l'organisation spatio-temporelle des séquences, les modalités de déplacement et de communication en classe de l'enseignant et des élèves, la nature des outils utilisés par l'enseignant et les élèves pour interagir (par ex. le recours à des pictogrammes pour symboliser la consigne de travail donné à l'élève) ou encore la nature du travail demandé aux élèves. Ces données d'observation n'ont pas fait l'objet d'une analyse en soi mais ont servi de support, en qualité de trace de l'activité des enseignants en classe, pour les entretiens.

Trois entretiens semi-directifs de type instruction au sosie (Odone et al., 1981) ont été réalisés à la suite de chacune des séquences d'enseignement ayant été observées par le chercheur. Ces entretiens ont pour objectif d'appréhender leur activité professionnelle en situation de travail. A partir de la consigne initiale inspirée de Clot et Soubiran (1998) « Suppose que je sois ton sosie et que demain je me trouve en situation de te remplacer dans ton travail. Je te questionne sur la manière dont je dois agir, en insistant sur les dêtails », le chercheur invitait l'enseignant à lui délivrer les instructions les plus précises possibles sur la manière de réaliser le travail. Par cette démarche, le chercheur vise à infiltrer le cœur de l'action de l'enseignant afin de l'accompagner dans l'explicitation de celle-ci. Les instructions au sosie ont été d'une durée variable allant de 23 à 57 minutes. Les trois entretiens ont été enregistrés à l'aide d'un dictaphone.

\section{Traitement des données}

Après avoir été retranscrit, le corpus des données d'entretien a été découpé en unités d'analyse en référence aux postulats théoriques retenus empruntés à une théorie de l'activité (Leontiev, 1976) ${ }^{1}$. Ces unités ont été délimitées à partir de l'identification dans les propos de l'acteur, d'abord, d'un but d'action, puis complété par le repérage des opérations et des motifs d'agir de l'acteur. Chaque unité a ainsi pu être formalisée de la manière suivante : [faire ceci (but)... en procédant ainsi (opérations)... parce que (motif)...]. Suivant la méthode du codage ouvert empruntée à la théorie ancrée (Strauss \& Corbin, 1990), les unités ont été analysées et comparées dans une démarche d'abstraction afin de les classer et d'extraire les savoirs professionnels des enseignants. Le Tableau 2 présente une illustration de la procédure de traitement des données.

Tableau 2 : Illustration de la procédure de traitement des données d'entretien.

\begin{tabular}{c|c}
\hline Entretien & Traitement \\
\hline 60 Max : Avec Dylan et Arnaud j'emploie moins de mots pour que & \\
\hline
\end{tabular}

\footnotetext{
${ }^{1}$ Pour Leontiev (1976) l'activité peut être définie comme les agissements d'un sujet en fonction de buts (ce qu'il cherche à réaliser dans la situation) indexés à la fois à des « mobiles vitaux » (ce qui le pousse à agir, les raisons profondes de son engagement) et à des « opérations » (ce qu'il met en œuvre concrètement pour agir en termes de techniques, de gestes professionnels).

Flavier, E., Clément, C. (2014). Besoins de formation des enseignants du second degré pour l'inclusion des élèves avec un trouble du spectre de l'autisme. Nouvelle Revue de l'Adaptation et de la Scolarisation, 65, 95-112.
} 
ce soit plus clair, qu'ils ne se perdent pas dans les mots

$61 \mathrm{Ch}$ : En lui disant «j'ai changé d'avis » vous prenez sur vous et cela coupe court à ...

62 Max : Oui et il accepte, il ne sera pas angoissé, il n’y a pas d'angoisse

$63 \mathrm{Ch}$ : Il va basculer sur la nouvelle consigne?

64 Max : Oui voilà tout à fait, bien qu'il soit angoissé quand il rentre dans un environnement nouveau, tout changement le perturbe, qu'on ait des TSA ou pas, mais heu, quand l'élève avec TSA se sent en confiance, les imprévus sont beaucoup plus facilement gérés

$65 \mathrm{Ch}$ : Donc il faut créer entretenir cette relation de confiance

66 Max : Et puis pourquoi je lui ai demandé d'écrire à Dylan parce qu'il a une écriture très écorchée pour le faire exercer, voilà on est le matin alors je peux demander plus au niveau écrit donc j'hésite pas

Demander à Dylan d'écrire tout le texte [en procédant ainsi] Annoncer «j'ai changé d'avis » [parce que] éviter l'angoisse de Dylan [et] faire progresser l'élève / son écriture écorchée

Par cette démarche méthodologique de traitement des données, nous cherchons à rendre intelligible l'activité des enseignants en situation de classe. En identifiant les éléments constitutifs de leur activité (but, mobile et opération), nous nous donnons les moyens d'accéder à une compréhension de cette activité telle qu'elle est vécue et perçue par les enseignants eux-mêmes.

\section{Résultats}

L'analyse des données quantitatives et qualitatives a permis de mettre en exergue le caractère fondamental des connaissances spécifiques aux TSA dans l'accompagnement en milieu scolaire, spécialisé ou non, d'élèves présentant de tels troubles.

\section{Données quantitatives}

En moyenne, les enseignants énoncent des connaissances faibles concernant les TSA, qu'il s'agisse des enseignants ayant accueilli des enfants avec un TSA $(M=1,40, E T=0,60)$ ou n'ayant jamais accueilli un enfant avec un TSA $(M=1,25$, ET=0,53). Ils rapportent des besoins de formation plutôt importants qu'ils aient accueilli ou non un enfant avec un TSA dans leur classe (respectivement $\mathrm{M}=3,91$, $\mathrm{ET}=1,66$ vs $\mathrm{M}=3,91$, $\mathrm{ET}=1,42$ ). Les enseignants ayant accueilli des enfants ou adolescents avec un TSA énoncent des retentissements en classe plus importants $(3,42, E T=1,39)$ que les enseignants n'en n'ayant pas accueilli $(M=3,36$, $\mathrm{ET}=1,63)$.

Si nous nous intéressons spécifiquement aux enseignants n'ayant jamais accueilli d'enfants avec un TSA (Tableau 3), nous constatons que les connaissances énoncées comme les plus faibles concernent les critères diagnostics $(M=1,10)$. Cependant dans l'ensemble les enseignants estiment avoir très peu de connaissances, quelles que soient les thématiques : et les besoins de formation énoncés sont importants et systématiquement supérieurs à 3,70 sur un maximum de 5 .

Tableau 3 : Moyennes sur une échelle de 1 à 5 (écart-types entre parenthèse) pour chacune des trois composantes explorées pour les enseignants n'ayant jamais accueilli d'enfant avec un TSA.

Flavier, E., Clément, C. (2014). Besoins de formation des enseignants du second degré pour l'inclusion des élèves avec un trouble du spectre de l'autisme. Nouvelle Revue de l'Adaptation et de la Scolarisation, 65, 95-112. 


\begin{tabular}{lllc}
\multicolumn{1}{c}{ Thématiques } & Connaissances & $\begin{array}{c}\text { Besoin de } \\
\text { formation }\end{array}$ & $\begin{array}{c}\text { Répercussion en } \\
\text { classe }\end{array}$ \\
\hline $\begin{array}{l}\text { Différence entre les différents } \\
\text { diagnostics de TED }\end{array}$ & $1,30(0,57)$ & $3,89(1,52)$ & / \\
\hline Critères diagnostics du DSM-IV-TR & $1,10(0,45)$ & $3,84(1,54)$ & $/$ \\
\hline Caractéristiques comportementales & $1,30(0,57)$ & $3,79(1,58)$ & $2,94(1,30)$ \\
\hline Troubles médicaux associés & $1,15(0,37)$ & $3,72(1,49)$ & $3,11(1,46)$ \\
\hline Troubles de la communication & $1,20(0,41)$ & $4,00(1,49)$ & $3,28(1,45)$ \\
\hline Problèmes liés à l'apprentissage & $1,50(0,78)$ & $3,84(1,34)$ & $3,56(1,50)$ \\
\hline Hypo et hypersensibilité sensorielle & $1,20(0,62)$ & $3,95(1,39)$ & $3,58(1,34)$ \\
\hline Déficit dans les interactions sociales & $1,30(0,57)$ & $4,00(1,41)$ & $3,67(1,46)$ \\
\hline Problèmes psychologiques & $1,65(0,81)$ & $4,00(1,41)$ & $3,83(1,25)$
\end{tabular}

Les analyses de corrélation montrent un lien légèrement significatif entre les connaissances et les besoins de formation $(r=0,17 ; p<0,05)$. Les enseignants veulent être formés sur les thématiques pour lesquelles ils ont le plus de connaissances. De plus, il existe une corrélation positive entre les connaissances et les répercussions supposées des thématiques en classe $(\mathrm{r}=0,25 ; \mathrm{p}<0,005)$. Plus les enseignants connaissent les thématiques, plus ils considèrent qu'elles ont des répercussions importantes en classe. Enfin, il existe une corrélation significative et positive entre les besoins de formation énoncés et les répercussions des thématiques en classe $(\mathrm{r}=0,39 ; \mathrm{p}<0,0001)$ : les enseignants souhaitent être formés sur des thématiques qu'ils jugent pertinentes en classe, c'est à dire celles qui sont supposées avoir le plus de retentissement.

Si les besoins de formation sont hiérarchisés, il est intéressant de noter que les thématiques liées aux apprentissages et les troubles de la communication ne sont pointées qu'au $4{ }^{\text {ème }}$ et $5^{\text {ème }}$ rang de cette hiérarchie, la $1^{\text {ère }}$ étant les problèmes psychologiques et la $7^{\text {ème }}$ et dernière étant les caractéristiques comportementales (Tableau 3 ).

Même si l'échantillon des quatre enseignants ayant accueilli un élève avec un TSA est faible, l'exploration des données est intéressante. Ainsi, il existe une corrélation négative et non significative $(\mathrm{r}=-0,29)$ entre les connaissances et les besoins de formation, et une corrélation positive significative entre les besoins de formation et la répercussion des thématiques en classe $(\mathrm{r}=0,7 ; \mathrm{p}<0,0001)$. Les enseignants ayant accueilli au moins un élève avec un TSA souhaitent eux aussi être formés sur des thématiques qu'ils jugent pertinentes en classe. Pour ces derniers une présentation de la hiérarchisation des thématiques apparaît peu pertinente compte tenu du faible nombre de données qui engendre de multiples ex-æquo dans la hiérarchie.

La population enseignante étant l'une des plus féminisées, les données pour chaque énoncé ont été comparées selon le genre. Le Tableau 4 présente les données statistiquement

Flavier, E., Clément, C. (2014). Besoins de formation des enseignants du second degré pour l'inclusion des élèves avec un trouble du spectre de l'autisme. Nouvelle Revue de l'Adaptation et de la Scolarisation, 65, 95-112. 
significatives. Sur les cinq comparaisons significatives, quatre portent sur le retentissement supposé en classe

Tableau 4: Synthèse des tests $t$ significatifs; comparaisons de moyennes pour échantillons indépendants (hommes vs femmes).

\begin{tabular}{lllllll}
\hline & Hommes & Femmes & t & ddl & $\mathrm{p}$ \\
\hline $\begin{array}{l}\text { Retentissement en classe des } \\
\text { caractéristiques comportementales }\end{array}$ & 2,71 & 3,76 & $-2,797$ & 19 & 0,011 \\
\hline $\begin{array}{l}\text { Retentissement en classe des } \\
\text { problèmes médicaux }\end{array}$ & 1,86 & 3,67 & $-3,455$ & 20 & 0,003 \\
$\begin{array}{l}\text { Connaissances sur les difficultés } \\
\text { d'apprentissage }\end{array}$ & 1,29 & 2,18 & $-2,525$ & 21 & 0,020 \\
$\begin{array}{l}\text { Retentissement en classe de l'hyper } \\
\text { ou l'hypo sensibilité sensorielle }\end{array}$ & 2,43 & 4,06 & $-2,729$ & 21 & 0,013 \\
$\begin{array}{l}\text { Retentissement en classe des } \\
\text { difficultés psychologiques }\end{array}$ & 2,43 & 4,06 & $-2,984$ & 21 & 0,007 \\
\hline
\end{tabular}

\section{Données qualitatives}

L'analyse de l'activité des deux enseignants spécialisés exerçant en ULIS confirme l'importance de disposer de connaissances précises pour une prise en charge efficace des élèves présentant des TSA. Complémentairement aux résultats quantitatifs précédemment exposés, cette analyse permet de caractériser plus finement la nature des connaissances mobilisées (Extrait 1).

\footnotetext{
Extrait 1 (entretien MB1)

$\mathrm{Ch}$ : Votre formation vous a apporté quels types de connaissances ?

Mary : Voir un petit peu quelles sont les façons dont cela se répercute au niveau du comportement et ensuite au niveau des apprentissages.

Ch: C'est-à-dire quelles implications ou quelles conséquences ou quelles adaptations ?

Mary : Ben, c'est un peu les deux, un peu les deux, maintenant on rentre pas dans les détails au niveau du médical, on a eu certains cours là-dessus, la façon dont ça se traduit. Ce n'est pas tant le côté médical mais plutôt les conséquences que cela peut avoir en termes de comportements, d'attention, de concentration... Voilà les choses auxquelles je vais être vraiment confrontée.
}

Aussi importantes qu'elles sont, les connaissances relatives aux TSA ne peuvent se limiter à la dimension médicale. Pour être utiles à l'enseignant, elles doivent d'être articulées avec les répercussions en classe, c'est-à-dire permettre à l'enseignant d'établir le lien entre les pathologies et leurs implications sur l'activité de l'élève.

La mobilisation des connaissances par les enseignants spécialisés est ainsi sous-tendue par deux processus : a) une relecture contextualisée des éléments de connaissances acquis en formation et b) la réalisation d'actions en apparente contradiction avec les connaissances sur les TSA.

Une relecture contextualisée des éléments de connaissances acquis en formation

Flavier, E., Clément, C. (2014). Besoins de formation des enseignants du second degré pour l'inclusion des élèves avec un trouble du spectre de l'autisme. Nouvelle Revue de l'Adaptation et de la Scolarisation, 65, 95-112. 
Si leur formation initiale leur a permis d'acquérir des connaissances spécifiques sur les TSA et les conséquences en classe, lorsqu'ils évoquent leur activité en classe, les deux enseignants le font en s'attachant à contextualiser et préciser les circonstances particulières des situations dans lesquelles ils sont engagés ainsi que les caractéristiques singulières de chacun des élèves. Ce niveau de précision est rendu possible par une démarche d'enquête, dans laquelle ils s'engagent, et qui vise à mieux connaitre les élèves pour agir avec eux. Ainsi, Max, à propos

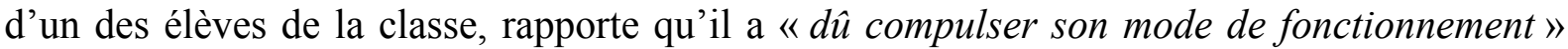
parce qu'il "vient d'arriver, c'est un primo entrant Alex» (Extrait entretien MN1). Mary confirme cette démarche de diagnostic avant de pouvoir intervenir avec justesse auprès des élèves : "je dirais qu'il n'y a pas vraiment de généralisation possible et qu'avant de pouvoir mettre en place des réponses qui vont être utiles aux élèves il faut bien identifier les besoins et comment on peut répondre à ces besoins » (Extrait entretien MB1).

Cette identification des besoins de chacun, vise à concrétiser les connaissances qư ils ont de la prise en charge des élèves présentant des TSA. En d'autres termes, il s'agit pour eux de passer d'énoncés génériques issus de leur formation et peu opérationnels à des connaissances actualisées et contextualisées à même de légitimer leurs choix en classe.

C'est ce qui se produit lorsque Max, au fil du temps et parce qu'il sait que les TSA s'accompagnent souvent de difficultés communicationnelles, construit une sorte de code langagier individualisé avec chacun des élèves afin d'être bien compris d'eux (Extrait 2).

\section{Extrait 2 (entretien MN1)}

Max : Dylan c'est tout ce qui est compréhension des consignes, heu, qui est à éclaircir, il faut vraiment que je sois très précis

Ch : Au début vous lui avez donné la consigne de tout recopier et de ne pas écrire sur la feuille, il dit " ah ça a changé », tout de suite vous lui dites "j'ai changé d'avis »

Max : Oui j'ai changé d'avis, il intègre assez bien les changements, les imprévus, heu, parce qu'il a l'habitude, alors j'utilise des mots assez précis, soit j'utilise « j'ai changé d'avis » ou « c'est annulé », pour Aude, voilà, j'utilise ce mot et puis on fait autre chose

Ch : C'est-à-dire qu'à chaque élève, il y a des petits mots comme ça, pour Aude, il y a «c'est annulé », pour David «j'ai changé d'avis »

Max : Oui, il y a des petits mots, et avec Aude je parle plus aussi, avec David et Arnaud surtout j'emploie moins de mots pour que ce soit plus clair, qu'ils ne se perdent pas dans les mots

Ch : En lui disant «j'ai changé d'avis » vous prenez sur vous et cela coupe court à toute

Max: Oui et il accepte, il ne sera pas angoissé

Tout acte d'enseignement implique pour l'enseignant de s'adapter, quasi individuellement, à l'élève pour lui permettre d'évoluer dans un environnement serein et propice aux apprentissages. Dans le cadre d'une scolarisation en ULIS, et particulièrement dans la prise en charge d'élèves présentant des TSA, il apparait nécessaire d'opérationnaliser ce principe. Cela passe d'abord par une enquête visant à identifier les canaux de réceptivité préférentiels de chaque élève, pour ensuite construire un code de communication dont l'efficacité tiendra notamment à la récurrence de son usage.

Cette actualisation des connaissances en situation de travail pour les enseignants dépasse le seul processus de contextualisation. Elle prend aussi la forme d'une reconsidération des acquis de la formation qui prennent alors un nouveau sens pour l'enseignant comme l'illustre l'Extrait 3.

Flavier, E., Clément, C. (2014). Besoins de formation des enseignants du second degré pour l'inclusion des élèves avec un trouble du spectre de l'autisme. Nouvelle Revue de l'Adaptation et de la Scolarisation, 65, 95-112. 


\begin{abstract}
Extrait 3 (entretien MB1)
Mary : j'ai été en début d'année en situation difficile parce que je me suis rendu compte que j'avais pas assez anticipé la chose par exemple avec Paul j'avais proposé une situation en géométrie où une des phases était de reproduire visuellement une figure à main levée. Je me suis rendu compte que Raphael a travaillé comme ça [mime l'élève travaillant avec une main en l'air, bras tendu vers le haut]. Je comprenais pas, je lui disais " tu as une question ? " il me répondait que « non ». C'est seulement après quand j'ai relu ma consigne que j'ai vu que c'était parce que j'avais écrit de reproduire la figure à main levée qu'il a travaillé comme ça [mime de nouveau l'élève]. Je l'ai mis en difficulté c'est une chose que je n'avais pas du tout anticipé

Ch: Les implicites ou les sens secondaires des expressions

Mary : Voilà, ça a été pris au premier degré. Ce qui va être facilitateur pour nous qui ne sommes pas dans le spectre ne va pas l'être pour eux donc il faut vraiment que notre seuil heu que l'on se dise «mais c'est pas pour moi » et donc pour lui « est-ce que cela va être facilitateur?»

$\mathrm{Ch}$ : Ce sont des choses déjà acquises en formation ou qui se sont actualisées

Mary : Ce sont des choses que l'on m'a dites en formation que j'ai notées mais en situation de travail que j'avais du mal au début à prendre en compte. Donc ce sont des choses que je savais mais que je n'arrivais pas encore à mettre en lien.
\end{abstract}

Dans le cadre de leur formation, notamment initiale, les enseignants spécialisés sont sensibilisés aux conséquences des TSA sur l'activité d'apprentissage des élèves. L'une d'entre-elles réside dans une compréhension littérale du langage altérant fortement les capacités de compréhension des consignes de travail par les élèves présentant des TSA. Pour autant, la connaissance par les enseignants de cet état de fait ne s'accompagne pas spontanément de l'intégration des réelles répercussions que cela recouvre pour leur manière d'intervenir en classe. Dans le cas présent, l'expérience vécue par Mary lui a permis de prendre la pleine mesure des difficultés que peuvent rencontrer les élèves dans la construction du sens des consignes relatives au travail scolaire.

Enfin, la dynamique de cette démarche d'enquête par l'enseignant ne s'inscrit pas seulement dans le cadre de la construction d'une relation personnelle avec chaque élève mais vise également à lui permettre de repérer les accointances particulières qui peuvent les lier. En d'autres termes, les situations conçues par l'enseignant permettent de dépasser les difficultés inhérentes au déficit dans les interactions sociales. Ainsi, "s'ils sont tous les deux ensemble c'est pas pour rien, ils s'aident mutuellement » affirme Mary à propos de Dan et Marc. Au cours des premiers mois de l'année, elle a procédé à de multiples essais dans l'organisation spatiale de la classe et le placement des élèves jusqu'à ce que « cela se stabilise depuis heu... début janvier [1'entretien a eu lieu en février]». Cet agencement, aujourd'hui pérenne s'appuie sur les caractéristiques de chacun : "en l'occurrence c'était de mettre Dan et Marc ensemble. Ensuite que Aline ait de la place à côté d'elle parce qu'elle s'étale beaucoup quand elle travaille, que Nico et Paul soient cachés de la fenêtre parce qu'il y avait trop d'éléments extérieurs qui pouvaient les parasiter, ensuite Tim est gaucher, donc s'il est dans un coin il dérange moins les autres, voilà c'est le petit genre de chose qui fait que pour eux ce soit moins perturbant» (Extrait entretien MB1). En agissant ainsi, plus que dans un environnement usuel, Mary s'attache à adapter l'environnement aux élèves pour compenser les difficultés engendrées par les TSA.

La réalisation d'actions en apparente contradiction avec les connaissances sur les TSA

Flavier, E., Clément, C. (2014). Besoins de formation des enseignants du second degré pour l'inclusion des élèves avec un trouble du spectre de l'autisme. Nouvelle Revue de l'Adaptation et de la Scolarisation, 65, 95-112. 
L'analyse de l'activité des deux enseignants spécialisés révèle, au-delà de cette contextualisation des connaissances par une démarche d'enquête, un processus de mise à distance de ces connaissances acquises en formation. Plus précisément, nos résultats montrent que les enseignants s'engagent dans des actions qui pourraient passer pour être en décalage avec les préconisations usuelles.

Ainsi, en dépit de l'efficacité reconnue des renforçateurs, notamment avec les élèves présentant des TSA, Max « repousse souvent l'utilisation de renforçateurs » (Extrait entretien MN1). Dans cette situation, l'enseignant s'affranchit donc volontairement des conseils et des techniques qui lui ont été dispensés et dont ses expériences passées lui ont prouvé qu'ils permettaient d'obtenir de bons résultats : "je constate que ça marche à 100\% quand on le met en place ». Pour autant, de telles actions ne sont pas fortuites. Elles sont sous-tendues par des motifs d'agir qui s'inscrivent dans un empan temporel plus long et qui répondent à des préoccupations tout à fait en adéquation avec les missions de l'enseignant. En confrontant l'élève à une autre forme de réalité, il s'agit pour Max de tester sa capacité d'adaptation et de "voir si un élève avec TSA est capable de s'en passer", "par souci de converger le plus vers la normalité entre guillemets ».

Mary défend les mêmes idées lorsqu'elle choisit de confronter les élèves de l'ULIS à des situations difficiles ou lorsqu'elle les amène à travailler dans les mêmes conditions que les élèves des autres classes. Il s'agit alors de les préparer à agir dans un environnement qui n'est ni spécialisé ni particulièrement adapté. En effet, si «L'inclusion c'est le monde qui doit s'adapter, dans la réalité, c'est pas comme ça, donc il faut aussi qu'ils [les élèves de l'ULIS] s'adaptent pour que les autres nous considèrent, ce n'est pas qu'aux autres de faire des efforts » (extrait entretien MB1).

\section{Discussion}

L'originalité de cette étude tient au regard croisé qu'elle propose dans l'identification des besoins de formation des enseignants du second degré concernant les TSA. Les résultats ont permis de mettre en évidence les besoins de formation perçus et déclarés par les enseignants du secondaire au moyen d'un questionnaire et de les confronter aux besoins de formation identifiés à partir de l'analyse de l'activité en classe de deux enseignants exerçant en ULIS et accueillant des élèves avec un TSA. Par cette démarche, nous ambitionnons d'approcher les besoins de formations réels des enseignants, c'est-à-dire de repérer la nature et le niveau des connaissances requises et leur adéquations avec les caractéristiques singulières des situations dans lesquelles elles sont mobilisées.

Les données recueillies à l'aide du questionnaire montrent que les connaissances autorapportées des enseignants sont faibles. Ceci rejoint les données de la littérature (Guimont et Forget, 2010), dans des proportions plus importantes cependant chez les enseignants n'ayant jamais accueilli d'élève avec un TSA. De plus ils estiment leurs besoins de formation comme importants afin de combler ce déficit, ce qui rejoint également de précédentes conclusions d'études chez des enseignants du primaire (Helps et al., 1999). Dès lors, disposer de connaissances relatives aux TSA pour accueillir efficacement des élèves présentant de tels troubles en classe apparait comme une condition première. Pour autant, les données du volet qualitatif permettent de nuancer quelque peu ces affirmations. En effet, il apparait, à la lumière de nos résultats que ce ne sont pas tant les connaissances sur les plans scientifique et médical qui priment, mais celles se rapportant aux conséquences sur l'activité en classe des élèves qui sont plébiscitées. Ainsi, tout comme dans l'étude de Guimont et Forget (2010), les enseignants évaluent avant tout leurs besoins de formation en lien avec les répercussions supposées en classe des thématiques, que ces enseignants aient accueilli ou non des élèves avec un TSA. Nous pointons cependant ici la difficulté d'estimer avec justesse ces Flavier, E., Clément, C. (2014). Besoins de formation des enseignants du second degré pour l'inclusion des élèves avec un trouble du spectre de l'autisme. Nouvelle Revue de l'Adaptation et de la Scolarisation, 65, 95-112. 
conséquences en l'absence d'une connaissance minimale des TSA et des difficultés qu'ils engendrent dans les activités d'apprentissage. En d'autres termes, une formation des enseignants à la prise en charge des TSA doit non seulement leur permettre d'acquérir des connaissances dites de base sur les différentes pathologies, mais également de les sensibiliser aux répercussions effectives de ces troubles sur l'activité des élèves en classe. Il est intéressant de noter ici que les femmes de notre échantillon anticipent des conséquences en classe plus importantes du trouble, sans que nous ne puissions clairement valider si ceci est généré par une anxiété plus importante ou une meilleure appréciation du trouble et de ses conséquences.

Si une formation doit tenir compte des besoins de formation énoncés, elle ne pourrait $s^{\prime} y$ résumer. En effet les thématiques hiérarchisées chez les enseignants n'ayant jamais accueilli d'enfants avec un TSA montrent que les caractéristiques comportementales, les troubles de la communication ou les problèmes liés à l'apprentissage ne sont pas priorisés. Or, l'une des premières difficultés en classe sera la difficulté à communiquer. D'un point de vue empirique, ceci est souligné par les deux enseignants interrogés. Cela rejoint également les conclusions de Helps et al. (1999) selon lesquelles les enseignants du primaire ayant accueilli des élèves avec autisme énonçaient comme principales difficultés leur manque de connaissances, mais aussi certains comportements associés à l'autisme comme les comportements d'agression et les difficultés de communication. Selon Humphrey et Symes (2011), ces altérations langagières et les difficultés à établir des relations sociales ou de collaboration avec des pairs sont des caractéristiques prégnantes chez les adolescents présentant des TSA. Corroborant les affirmations de Haye et Courtinat-Camps (2013), l'analyse de l'activité en classe des enseignants exerçant en ULIS confirme cet état de fait en ce sens que tous deux ont indiqué faire preuve d'une attention soutenue à l'établissement d'un climat propice aux échanges en classe. Plus précisément, dans leurs actions et leurs propos, les enseignants s'attachent à l'élaboration de codes de communication précis et parfois même individualisés. Parmi leurs préoccupations, celle de se faire correctement comprendre par les élèves occupe une place significative. Cela s'explique notamment par la difficulté qu'ils ont à comprendre le sens non littéral du langage, les métaphores, le second degré (Beaud, 2011). A cette fin, ils recourent fréquemment à l'usage de stimuli discriminatifs (par exemple des illustrations, des mots clés ou des pictogrammes). Comme ont pu le montrer Odier-Guedj et Gombert (2012), le recours à des médiateurs pour compléter une consigne orale constitue un puissant levier d'aide à la compréhension de cette dernière.

Avoir des connaissances théoriques sur les TSA est une première étape pour comprendre les besoins et les modalités de fonctionnement des élèves, mais cela ne peut suffire pour prétendre intervenir efficacement en classe. Le volet qualitatif des résultats de notre étude met particulièrement en exergue le caractère singulier et localement adapté de l'activité des enseignants. Ces derniers identifient leurs connaissances moins par des énoncés génériques que par l'adossement à des situations particulières et bien délimitées. Nous en retenons que la démarche d'enquête dans laquelle ils s'engagent, principalement en début d'année, est un passage incontournable à la construction de ces connaissances situées et incarnées dans le contexte de la classe.

Avec Grimaud (2012), nous soutenons l'idée que l'activité des enseignants en milieu spécialisé s'appuie avant tout sur la mobilisation de " gestes ordinaires » (p. 175), pour peu que ces derniers ne consistent pas en l'application stricte de techniques apprises en formation et de manière décontextualisée. Ainsi, à notre sens, une formation à l'accompagnement d'élèves avec des TSA ne devrait pas s'attacher à dispenser des techniques professionnelles comme autant de " prêt-à-agir » de l'intervention éducative mais gagnerait à faire émerger et construire ces connaissances à partir de la transformation et l'analyse de pratiques réelles. En Flavier, E., Clément, C. (2014). Besoins de formation des enseignants du second degré pour l'inclusion des élèves avec un trouble du spectre de l'autisme. Nouvelle Revue de l'Adaptation et de la Scolarisation, 65, 95-112. 
d'autres termes, nous défendons une formation qui s'adosse aux expériences individuelles vécues. Ainsi concevoir une pédagogie à la prise en charge des élèves présentant des TSA ne peut s'envisager à vide ; l'inscription de la réflexion dans un contexte réel d'enseignement devient une nécessité. Dans le cadre d'une formation initiale ou continue, il est alors possible de penser des dispositifs s'inscrivant dans un empan temporel «long » (de quelques semaines à quelques mois) permettant des allers retours entre les temps de formation et les temps d'activité professionnelle (sous la forme de stage lorsqu'il s'agit d'une formation initiale). En s'adossant à des moments d'analyse de l'activité professionnelle des enseignants, notamment sur la base d'enregistrements vidéo de séquences d'enseignement, la mise en exergue de la démarche d'enquête et de l'adaptation progressive des méthodes pédagogiques mises en œuvre prend tout son sens. Complémentairement, le recours à des récits de vie ou à des témoignages d'expériences vécues (par ex. Haye et Courtinat-Camps, 2013) constituent des supports de formation tout aussi heuristiques et moins lourds que les documents vidéo.

\section{Conclusion}

Cette recherche exploratoire visait à l'identification des besoins de formation des enseignants du second degré par la confrontation entre les besoins perçus qu'ils déclarent et les besoins réels qui émergent de l'analyse de leur pratique professionnelle. Les données quantitatives montrent un faible niveau de connaissances sur les TSA par les enseignants du second degré, qu'ils aient accueilli ou non un élève avec ce type de troubles. En cohérence avec de précédentes études, les enseignants souhaitent être formés sur des thématiques ayant selon eux des retentissements importants en classe. Les données qualitatives viennent confirmer ce besoin comme une nécessité permettant une prise en charge ad hoc de l'élève présentant des TSA. Des connaissances précises sur les conséquences des TSA sur l'activité en classe des élèves sont mobilisées par les enseignants pour penser et réaliser leur enseignement. Néanmoins, nos données montrent également que ces connaissances ne suffisent pas et se révèlent peu opérationnelles dès lors qu'elles ne donnent pas lieu à une enquête circonstanciée permettant leur adaptation aux conditions singulières du contexte d'intervention.

Cette recherche exploratoire ouvre des perspectives tant pour la conception de la formation initiale que continue des enseignants du second degré. En particulier l'articulation des volets quantitatifs et qualitatifs permet d'interroger la pertinence de modules d'enseignement dédiés strictement aux TSA. Même si une connaissance minimale des TSA est sans doute nécessaire pour l'enseignant avant toute inclusion, la connaissance des ressources disponibles dans son milieu et la mise en ceuvre d'un dispositif de formation continue dédié à l'ensemble des enseignants accueillant des élèves avec un TSA, sont des pistes à ne pas négliger, pour être au plus près des besoins des enseignants.

Cependant le nombre limité de répondants ne permet pas de discuter certains éléments plus avant. Il s'avère ainsi nécessaire de poursuivre le recueil des données pour explorer davantage les différences concernant les besoins de formation perçus entre enseignants ayant ou n'ayant jamais accueilli un élève avec un TSA.

\section{Références bibliographiques}

Beaud, L. (2011). Sur la littéralité autistique : Langage et communication sociale à l'épreuve de la métaphore. Glossa, 110, 26-48.

Beauguitte, A. (2006). Étude sur l'intégration scolaire des enfants autistes en France. Paris : Autistes sans frontières.

Conseil Economique Social et Environnemental (2012). Le coût économique et social de l'autisme. Avis du Conseil Economique Social et Environnemental. Paris : Journal officiel de la République Française.

Flavier, E., Clément, C. (2014). Besoins de formation des enseignants du second degré pour l'inclusion des élèves avec un trouble du spectre de l'autisme. Nouvelle Revue de l'Adaptation et de la Scolarisation, 65, 95-112. 
Campion, C.-L. \& Debré, I. (2012). Rapport d'information au nom de la commission sénatoriale pour le contrôle de l'application des lois (1) sur l'application de la loi $\mathrm{n}^{\circ}$ 2005-102 du11 février 2005, pour l'égalité des droits et des chances, la participation et la citoyenneté des personnes handicapées. Paris : République Française.

Clot, Y. \& Soubiran, M. (1998). Prendre la classe : une question de style ? Société française, $12 / 13(62 / 63), 78-88$.

Dillon, G.V. \& Underwood, J.D.M. (2012). Parental Perspectives of Students With Autism Spectrum Disorders Transitioning From Primary to Secondary School in the United Kingdom. Focus on Autism and Other Developmental Disabilities, 27, 111-121. DOI: $10.1177 / 1088357612441827$

Ensemble pour l'autisme (2012). http://www.autismegrandecause2012.fr/fr/autisme-enfrance.html, récupéré le $1^{\mathrm{er}}$ août 2013.

Grimaud, F. (2012). Coopération entre chercheur et enseignants scolarisant un élève en situation de handicap : une co-activité. La nouvelle revue de l'adaptation et de la scolarisation, 57, 167-180.

Guimond, F.A. \& Forget, J. (2010). Evaluation des connaissances et des besoins de formation d'enseignants au collégial sur les troubles envahissants du développement. Revue Francophone de la Déficience Intellectuelle, 21, 71-79.

Haute Autorité de Santé (Mars 2012). Autisme et autres troubles envahissants du développement : interventions éducatives et thérapeutiques coordonnées chez l'enfant et l'adolescent. http://www.has-sante.fr/portail/jcms/c_953959/autisme-et-autrestroubles-envahissants-du-developpement-interventions-educatives-et-therapeutiquescoordonnees-chez-lenfant-et-ladolescent

Haye, I., \& Courtinat-Camps, A. (2013). Pratique pédagogique en Unité d'Enseignement pour élèves autistes : Témoignage d'une enseignante spécialisée. La Nouvelle Revue de l'Adaptation et de la Scolarisation, 60 (numéro thématique : l'autisme, une grande cause scolaire), 157-167.

Helps, S., Newsom-Davis, I.C. \& Callias, M. (1999). Autism : The teacher's view, Autism, 3, 287-298. doi: 10.1177/1362361399003003006

Hendricks, D.R. (2007). A descriptive study of special education teachers serving students with autism: knowledge, practices employed and training needs (Doctoral dissertation, Virginia Commonwealth University, 2007).

Humphrey, N., \& Symes, W. (2011). Peer interaction patterns among adolescents with autistic spectrum disorders (ASDs) in mainstream school settings. Autism, 15(4), 397-419. doi: $10.1177 / 1362361310387804$

INSERM Expertise collective (2002). Troubles mentaux. Dépistage et prévention chez l'enfant et l'adolescent. Paris : Editions INSERM.

Johnson, R.B. \& Onwuegbuzie, A.J. (2004). Mixed methods research: a research paradigm whose time has come. Educational Researcher, 33(7), 14-26.

LOI n 2005-102 du 11 février 2005 pour l'égalité des droits et des chances, la participation et la citoyenneté des personnes handicapées. Journal Officiel de la République Française, $2353 . \quad$ Récupéré de http://www.legifrance.gouv.fr/affichTexte.do?cidTexte=JORFTEXT000000809647\&d ateTexte $=\&$ categorieLien $=$ id le $1^{\circ}$ août 2013 .

Juhel, J.C. (2005). L'intégration au collégial d'étudiants ayant un syndrome d'Asperger. Revue Québécoise de Psychologie, 26, 239-255.

Leontiev, A. (1976). Le développement du psychisme. Paris : Editions sociales.

Flavier, E., Clément, C. (2014). Besoins de formation des enseignants du second degré pour l'inclusion des élèves avec un trouble du spectre de l'autisme. Nouvelle Revue de l'Adaptation et de la Scolarisation, 65, 95-112. 
Ministère des Affaires sociales et de la Santé (Mai 2008). Plan Autisme 2008-2010. Récupéré de www.sante.gouv.fr/IMG/pdf/Plan_autisme_2008-2010.pdf le $1^{\circ}$ août 2013.

Odier-Guedj, D. \& Gombert, A. (2012). Pratiques collaboratives en classe : amélioration de la compréhension des consignes chez des élèves dyslexiques et autistes. La nouvelle revue de l'adaptation et de la scolarisation, 57, 117-130.

Odone, I., Rey, A., \& Briante, G., (1981). Redécouvrir l'expérience ouvrière. Vers une autre psychologie du travail. Paris : Editions Sociales.

Organisation Mondiale de la Santé (1994). Classification internationale des troubles mentaux et des troubles du comportement. Descriptions cliniques et directives pour le diagnostic. Paris, France : Masson.

Paquet, A., Clément, C., \& Magerotte, G. (2012). L'inclusion scolaire des enfants âyant un trouble envahissant du développement : évolution des services et défis de trois régions de la francophonie. In. C. Philip, G. Magerotte, J.L.- Adrien (Eds). Scolariser des élèves avec autisme et TED. Paris : Dunod.

Flavier, E., Clément, C. (2014). Besoins de formation des enseignants du second degré pour l'inclusion des élèves avec un trouble du spectre de l'autisme. Nouvelle Revue de l'Adaptation et de la Scolarisation, 65, 95-112. 